\title{
Development and Pilot Testing of a Novel Graphic Values History Tool To Support Communication and Decision Making During Serious Illness
}

Peter Allatt, MA MHSc and John J. You, MD MSc

About the Authors:

Peter Allatt, MA MHSc is with Bridgepoint Active Healthcare, Sinai Health System, Toronto, Ontario.

John J. You, MD MSc, is with the Departments of Medicine, and Health Research Methods, Evidence, and Impact, McMaster University, Hamilton, Ontario.

Corresponding Author:jyou@mcmaster.ca

Submitted: July 23, 2018. Accepted: October 3, 2018. Published: May 21, 2019. DOI:10.22374/cjgim.v14i2.301

\section{ABSTRACT}

\section{Background}

We report the development and pilot testing of a values history tool to support patients and their substitute decision makers (SDMs) in advance care planning.

\section{Methods}

Tool development was an iterative, multi-phase process involving expert input, literature review, patient input, and beta testing with 35 laypersons. In a single-centre, before-after pilot study, we assessed the acceptability, clarity, and potential utility of the tool.

\section{Results}

Participants ( $\mathrm{n}=6 ; 3$ patients and 3 SDMs) rated the tool as clear (4.7/5), worthwhile (4.7/5), and said it did not cause distress (4.7/5). Tool completion and discussion resulted in a better understanding of patient values (4.5/5) and helped build understanding between patient and $\operatorname{SDM}(4.3 / 5)$.

\section{Conclusions}

Based on development involving content experts, patients, and laypersons, we conclude that our tool has face and content validity. Our pilot study suggests it merits evaluation in larger studies to further evaluate its utility.

Keywords: values; advance care planning; end-of-life care; shared decision making; medical decision making 
Too often, patients receive end-of-life care that is inconsistent with their values and preferences. ${ }^{1,2}$ Indeed, patients who have a serious illness, and their families, have indicated that improvements in end-of-life communication and decision making are a top priority for improving the quality of end-of-life care. ${ }^{3}$ End-oflife communication and decision making involve several related but distinct activities: advance care planning (ACP), goals-ofcare discussions, and in-the-moment decision making about treatments. ${ }^{4}$ Values form the foundation of all of these processes (Figure 1). ACP is "a process that supports adults at any age or stage of health in understanding and sharing their personal values, life goals, and preferences regarding future care." 5 The goal of $\mathrm{ACP}$ is to help ensure that people receive future medical care that is consistent with their values by preparing substitute decision makers (SDMs) to make future "in-the-moment" decisions on the patient's behalf. It is not exclusively for end-of-life and is ideally initiated before a person develops serious illness. It does not constitute consent to treatment. ${ }^{6}$ Goals-of-care discussions take place in the context of a serious, life-limiting illness. They are conversations between clinicians and patients with serious illness, or their substitute decision makers (SDM), about the patient's understanding of their illness and prognosis, and about the patient's values and priorities related to health care. Once again, the aim of a goals-of-care discussion is to ensure that "in-the-moment" decisions about treatment are aligned with patients' values and goals.

In this paper, we define values as "things that are important to the patient." Values exert a major influence on a patient's decisions about treatment and may include the acceptability of different health states, the impacts of illness on relationships with family and friends, and the influence of religious and cultural beliefs. Values may change over time. Although decisions about care during serious illness should be guided by a patient's values, ${ }^{8,9} \mathrm{SDM}$ are often poorly informed about patient values, ${ }^{10}$ clinicians often do not ask patients about their values, ${ }^{11}$ and patients themselves frequently make value statements that are internally inconsistent. ${ }^{12}$ Values history tools may offer a solution to these problems.

A values history tool is an instrument that can help a patient explore, clarify and articulate his or her values, and that can stimulate conversation with SDMs or health care providers. ${ }^{13-18}$ The original values history was a series of open-ended questions that allowed a patient to describe what was important to him or her ${ }^{16}$; over time, values histories evolved to include rating scales in place of open-ended questions and have been incorporated into formal decision aids. ${ }^{19,20}$ However, early tools were often too complex, too narrowly focused on specific medical conditions, and too dependent on facilitation by (time-strapped) physicians (Table 1).

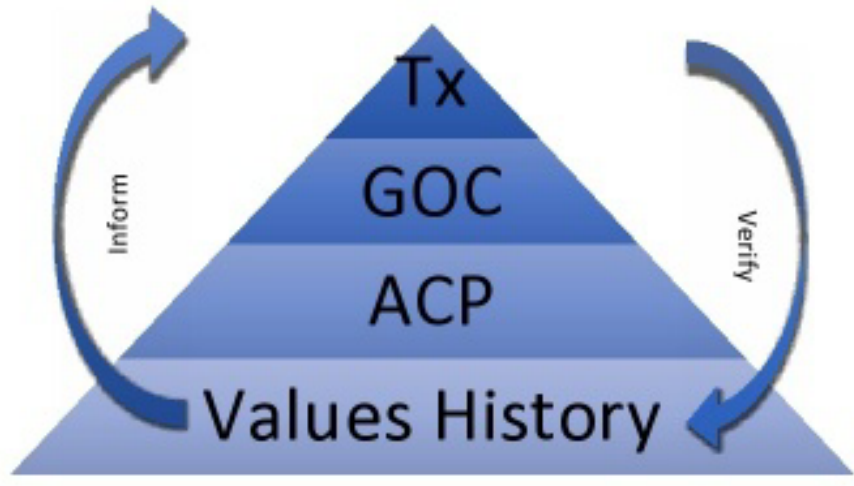

Figure 1. Patient Values are the Foundation of Serious Illness Communication and Decision-Making. Ascending the values pyramid from general to specific, patient values inform all activities. In turn, patient values can be used to verify (concordance check) choices or activities when descending the pyramid. For example, the SDM can use patient values to confirm that an "in-the-moment" patient care decision is in keeping with the patient's wishes, beliefs and preferences. Abbreviations: Tx, "in-themoment" treatment decisions; GOC, goals-of-care discussions; ACP, advance care planning.

The National Framework document for the Advance Care Planning in Canada initiative states that "in order for professionals and the public to engage in ACP they require a number of tools ... [that] must be innovative and in plain language."21 Accordingly, we set out to develop a values history tool that addressed the limitations of earlier tools and that could be used by patients and their future SDMs to help them with ACP. This paper describes the development and pilot testing of this new tool, called the "Graphic Values History Tool" (GVHT).

\section{Methods}

\section{Design}

We conducted a single-centre, before-after pilot study to obtain a preliminary exploration of whether completion of the GVHT by patient and SDM dyads is associated with an improvement in the

Table 1. Limitations of Early Values History Tools

\begin{tabular}{|c|c|}
\hline Limitation & Solution \\
\hline Tools too complex, academic & Low literacy tool (plain language) \\
\hline $\begin{array}{l}\text { Tools require significant medical } \\
\text { knowledge }\end{array}$ & $\begin{array}{l}\text { Low health literacy tool (plain } \\
\text { language) }\end{array}$ \\
\hline $\begin{array}{l}\text { Tools designed for specific health } \\
\text { problems (e.g., cancer, HIV, stroke, } \\
\text { PVS) What happens if something else } \\
\text { presents? }\end{array}$ & $\begin{array}{l}\text { Flexible tool applicable to many } \\
\text { situations } \\
\text { Focus on impact on patient } \\
\text { values, beliefs, and fears }\end{array}$ \\
\hline Completion process driven by MD & Process driven by patient/SDM \\
\hline $\begin{array}{l}\text { MD has limited time to facilitate } \\
\text { process }\end{array}$ & Process driven by patient/SDM \\
\hline
\end{tabular}

HIV = human immunodeficiency virus; $\mathrm{MD}=$ medical doctor; PVS = persistent vegetative state; $\mathrm{SDM}=$ substitute decision maker. 
SDM's understanding of the patient's values. Secondary objectives were to determine: (1) whether completing the GVHT improves the patient's understanding of their own values and preferences; (2) whether completing the GVHT can be done without assistance; (3) whether a values history discussion between patient and SDM (i.e., after they each complete the GVHT independently) can be done without assistance; and 4) which format patients and SDMs prefer for completing the GVHT (paper or electronic).

\section{Setting and Participants for the Pilot Study}

From December 2013 to April 2014, we evaluated the GVHT at Bridgepoint Active Healthcare, Toronto, Ontario, Canada. Patients were eligible to participate in this study if they were inpatients or outpatients at our rehabilitation or complex continuing care hospital. This unfunded study received Joint Research Ethics Board approval. All study participants gave written informed consent.

\section{Development of the Graphic Values History Tool (GVHT)}

In December 2012 a multi-disciplinary team comprising a clinical ethicist, a palliative care physician, and 2 critical care physicians developed a prototype values history tool informed by their clinical expertise and the literature. ${ }^{14,15,17}$ The tool had a patient version and an SDM version, each posing 36 questions grouped in 4 sections: "exploring values," "exploring value conflicts," "are some conditions worse than death?" and "how do you weigh the odds of survival?" We obtained feedback from a convenience sample of 15 adult, English-speaking patients about the tool's readability, clarity, completeness, ease of use, and ability to facilitate decision making.

Feedback highlighted a need to more fully recognize that many patients have low literacy or low health literacy, and to recognize that some patients are visual learners. Accordingly, in the summer of 2013, we undertook a substantive revision to create a new tool called the GVHT for evaluation in the current study. These modifications included revisions to wording to increase clarity for a lay audience and the introduction of graphics. New graphic elements included a contextual image with each question; a 3-colour (red, white, and green) scale to help readers interpret response options on the 5-point Likert scale; a line with 2 anchors to depict trade-offs (e.g., between quality and quantity of life); and pie charts to illustrate percentages. Staff in Augmentative Communications and the Clinical Library at Bridgepoint Active Healthcare assisted with editing text and creating graphics. After an initial draft of the GVHT was created, we used an iterative process to make serial revisions to the tool based on suggestions for improvement from 35 beta testers who represented a wide range of potential end-users (Table 2). During this iterative process, beta testers were given the tool and asked to provide feedback on: (1) the
Table 2. Characteristics of 35 Individuals Who Beta-Tested Draft Versions of the Graphic Values History Tool

\begin{tabular}{|l|l|}
\multicolumn{1}{|c|}{ Characteristic } & \multicolumn{1}{c|}{ Range } \\
\hline Age & $20-93$ \\
\hline Education level & Grade school - postgraduate \\
\hline Health literacy & $\begin{array}{l}\text { Weak (no education) - Strong } \\
\text { (physician, nurse) }\end{array}$ \\
\hline Occupation & $\begin{array}{l}\text { Businessperson, artist, writer, nurse } \\
\text { (retired), spiritual care provider, } \\
\text { researcher, students, health care } \\
\text { providers, stay at home parent, } \\
\text { informal caregiver, allied health } \\
\text { professionals, medical students }\end{array}$ \\
\hline Health status of tester & Healthy - multiple co-morbidities \\
\hline Role & Patient and SDM or potential SDM \\
\hline
\end{tabular}

SDM = substitute decision maker.

clarity and readability of the introductory section of the tool, the section instructions, and the individual questions (to guide this feedback, beta testers were told that the goal was for the tool to use clear and accessible language); (2) the appropriateness of the graphics and their usefulness in helping to understand the questions; and (3) questions to be added or eliminated. As soon as we received substantive feedback from beta testers, we updated the tool. The next beta testers received the updated tool. This iterative process of beta testing continued until we received no further substantive feedback.

The final GVHT included the following features: ${ }^{19}$ it was available in both paper and electronic (computer or tablet-based) versions of the tool for patients (Appendix A) and for SDMs (Appendix B). The content of paper and electronic versions was identical. The tool comprised 52 questions in 8 sections: (1) Quality of Life - Independence; (2) Quality of Life - Medical Condition; (3) Value Conflicts - Part 1; (4) Value Conflicts Part 2; (5) Are Some Conditions Worse Than Death? (6) How Do You Weigh Chances Of Survival? (7) Impact of Decisions on Others; (8) Religious / Spiritual / Cultural Beliefs. The tool uses a rating method to elicit closed-ended responses about the importance of different values on a 5-point Likert scale or to quantify trade-offs between different values using a linear scale; however, it also includes open-ended questions after each closed-ended question so that respondents could contextualize their response using free text comments.

\section{Study Procedures}

Interested patients or SDMs were identified through initial contact by the patient care managers or social worker on participating units or through a recruitment poster. Research 
staff then approached interested individuals for enrollment in the study. To minimize discussion of values between patient and SDM until both had independently completed the tool, it was necessary for them to be available at the same time. At baseline, both parties were asked "How well do you understand your / the patient's health care values and preferences?" with the following response options: Very Poorly, Poorly, Uncertain, Well and Very Well. Each participant in the dyad then completed the respective values history tool independently. Patient and SDM versions were identical, except that the SDM version asks SDMs to consider the patient's values and preferences rather than their own.

After completing the tool, the patient and SDM reviewed each other's answers and discussed areas of concordance or discordance. At the end of the discussion, the patient could decide whether to alter his or her responses and update the document. During the discussion, the researcher was physically present but minimally involved as a facilitator, (available to answer questions) but as much as possible allowing patient and SDM to direct their own discussions.

Following the discussion, both patient and SDM were asked again to answer the question "How well do you understand your / the patient's health care values and preferences?" using the same response options as for the baseline question. Finally, both members of the dyad independently completed a standardized evaluation of the tool, the process of tool completion, and discussion. The evaluation instrument (Appendix C) asked 34 questions on a 5-point Likert scale (5=strongly agree to $1=$ strongly disagree). Questions addressed the clarity, completeness, utility, and acceptability of the introduction to values histories, the introduction to the tool, section instructions, graphics, process of completion, the tool in its entirety, and the facilitated discussion.

\section{Statistical Analysis}

Results are presented as counts for the primary question of "How well do you understand your / the patient's health care values and preferences?" or mean scores for the responses to questions evaluating the tool and the process of the tool's implementation.

\section{Results}

We enrolled 6 participants ( 3 patient/SDM dyads). Participants ranged from 51 to over 70 years of age. One SDM was male; all other participants were female. One participant had some high school education, 2 had some college education, one had a bachelor's degree and 2 had master's degrees. Two patients were outpatients, one was an inpatient; all had experienced one or more serious life events. One SDM was a spouse, one the adult child of the patient, and one was the legally appointed substitute decision maker (Power of Attorney for Personal Care). There were no consent refusals or study withdrawals.

\section{Impact on the Understanding of Patient Values}

When asked: "How well do you understand the patient's values and preferences" (primary outcome), one SDM answered "well" and 2 SDMs answered "very well," both at baseline and after completing the GVHT. One participant wrote, "We were pretty much in sync; have had these discussions before." (SDM 3) Although SDM understanding of patient values remained unchanged after completing the GVHT based on responses to this question, participants nonetheless said that the completed values history resulted in a better understanding of patient values (mean 4.5/5) (Table 3), and that the tool and discussion process helped identify differences between patient values and SDM understanding of patient values (mean 4.3/5). Responses to 3 questions elicited the advantage of having the values discussion: the document helped build understanding between the patient and SDM (mean 4.3/5); and helped resolve disagreements regarding patient values (mean 3.5/5). One commented, "I was a little more negative in my responses than I would have expected to be?" (SDM2) The GVHT also allowed patients to express their values. During discussion, for example, one patient said it was important to be clear-minded, able to spend time with her family, and not to be a burden on her family: "It depends how far away I was for the kids to come and see me, but if my mind was ok it would be ok but I don't want strain on the family." (P1) Another patient wrote that, "All of my answers will be based on degree of mobility. I think, that's how I went through it, I kind of thought, OK, will I be able to move, will I be able to think?" (P2) The oldest patient wrote that, "In terms of the quality of life and the quantity of life I definitely want the quality because I, at my age, do not feel that my quantity of life is very important." (P3) Finally, patients' understanding of their own values and preferences improved after completing the GVHT.

\section{Participant Evaluation of GVHT}

Participants evaluated the tool in its entirety and the process used to implement the tool (see Table 3). The tool was found to be clear, useful when thinking about patient values, and comprehensive. Pictures were deemed clear, easy to understand (mean 4.8/5) and sufficiently large (mean 4.8/5). One participant wrote: "Colour helpful! I was influenced by the symbol for pain very powerful." (SDM 2) Another said that the pictures were "not always necessary but can see how would be useful for people less familiar with the issue." (SDM 3) Both patients and SDMs found the document "good as a stand-alone tool" or as a "companion to other tools." While participants agreed that "completing the values history was thought provoking" (mean 4.7/5), they nonetheless said that completing it "did not cause distress" (mean 4.7/5).

One objective of this study was to obtain participant feedback about the process used to implement the tool, specifically whether tool and subsequent discussion could be completed 
Table 3. Participant ( $n=6)$ Evaluation of the Graphic Values History Tool

\section{Question}

Mean Score*

1. Values history tool was:

a. Easy to understand

4.3

b. In logical order

4.8

c. Useful in thinking about patient values

4.8

d. About values important to the patient

4.8

e. Easy to read

4.7

f. Did not leave out values important to the patient

2. The instructions for each section were:
a. Clear
b. Complete
4.6
4.6

3. The pictures were
a. Clear
4.7
b. Easy to understand
4.7
c. Large enough to see
4.8
d. Helpful in understanding the question

4. The use of colour in the Likert scales

a. Made it easier to understand the question

4.5

b. Helped make answers clear

4.7

c. Matched the answers

5. Completing the values history

a. Was a worthwhile exercise

4.7

b. Was thought provoking

c. Did not cause distress

d. Did not cause confusion

e. Was a good use of my time

6. The completed values history

a. Is a clear summary of patient values

b. Resulted in a better understanding of patient values than before the values history was completed

c. Helped identify differences between patient values and Substitute Decision Maker

d. Helped focus the discussion of values between patient and Substitute Decision Maker

e. Would be useful when making treatment decisions

f. Could be completed without assistance

g. Covered the values important to the patient

h. Was easy to complete in the format I chose (paper, tablet, laptop)

7. Introduction to the values history process

a. Provided me with a clear understanding of the process

b. Clearly indicated the focus was the patient's values

8. Facilitated discussion

a. Helped increase the understanding between the patient and SDM

b. Helped to resolve disagreements regarding patient values

c. Could be completed without assistance

d. Was a worthwhile addition to the process

* Responses were on a 5-point Likert scale: 1 = strongly disagree; 5 = strongly agree. 
without assistance by patient and SDM, and whether a paper or electronic version was preferred. Participants reported that the GVHT could be completed without assistance (mean 4.2/5), although one participant made the following comment: "Might depend on the age/health of patient" (P2). Another objective was to explore whether the discussion could be held without assistance. In the discussion, patients and SDMs discussed their responses to each question; if there was concordance in responses (patient and SDM had the same scores), they moved to the next question. Whenever there was minor or major discordance, the patient and SDM discussed their responses in more detail and provided context. Most of the time differences were clarified and concordance reached. When asked if the discussion could be completed without assistance, participants responded positively (mean 4.2/5). "Might be useful to have a third party facilitate discussion between patient and SDM," was one comment. (P2) Finally, with respect to a preferred format for completing the GVHT, although laptop, tablet, and print versions were made available, all participants chose the print format.

\section{Discussion}

In this paper, we report the development and initial evaluation of a novel values history tool to support ACP. Because development of the tool was based on input from clinical experts, the published literature, and substantial beta testing with end-users, we believe that the GVHT has both face and content validity. Our pilot study provides preliminary evidence that the GVHT may be feasible and acceptable for use by patients and their SDMs, may improve understanding of patient values, and suggests that further evaluation of the tool in a larger study is warranted.

The GVHT addresses some of the limitations of previously developed values history tools. ${ }^{13-18}$ First, earlier tools were best suited to individuals with greater health literacy. To develop the GVHT, we obtained input from people with diverse educational and occupational backgrounds, including those with lower health literacy, to maximize its clarity in terms of both content and format. Second, several previously developed tools were designed for individuals with specific health problems (e.g., cancer, stroke, etc.), thus limiting their broader applicability and uptake. In contrast, the GVHT asks users to consider a wide range of value statements that are not specific to any one health condition but that can be very informative as the patient or SDM faces future "in-the-moment" decisions about treatment during serious illness. Finally, since earlier tools were intended to be completed through a physician-dependent process, physicians' lack of time is a potential barrier to the uptake of these tools. We designed the GVHT so that a patient and their SDM could complete the tool independently, and then discuss their responses without physician facilitation.
Further, our concern that some participants might be upset by either the tool or the values discussion proved unwarranted: participants reported that the questions and discussion were thought provoking and that they did not cause distress. This finding is consistent with work that we and others have done in the area of ACP and end-of-life communication, showing that open communication about the possibilities associated with serious illness and dying can increase patient and family satisfaction, and reduce stress and anxiety. ${ }^{22-24}$

Although our sample is small and our findings require further confirmation, our study suggests that it may be feasible for the GVHT to be completed by patients and SDMs as an initial step in the ACP process, without the need for physician facilitation. Fundamental to ACP is that patients are clear about what matters most to them (i.e., their values) and that they communicate these values to their future SDM(s). We found that the GVHT helped patients and SDMs to think about and clarify their values and that the values discussion helped them to articulate these values. Accordingly, we hypothesize that after completing and discussing their responses to the GVHT, patients and their SDMs may be more prepared for subsequent conversations with clinicians about ACP, goals-of-care discussions, or "in-the-moment" decisions about medical treatments during serious illness, because they will have a deeper and shared understanding about patients' values.

Strengths of this study include a rigorous process of tool development, including appreciable input from experts, and beta testing with potential end-users from a broad mix of educational backgrounds. Our pilot study has some limitations. First, the sample size was small due to several challenges to recruitment including the unfunded nature of the study and the lack of availability of participants in a clinical setting (we required simultaneous availability of patient and SDM for at least 2 hours to complete the GVHT independently, subsequent discussion between patient and SDM, and completion of evaluation instruments). In addition, the generalizability of our pilot study findings may be limited due to the single-centre nature of the evaluation and the relatively high educational level of the participants. However, the goal of our pilot study was to conduct a preliminary field evaluation of the GVHT in a clinical environment to better understand its acceptability, clarity, and potential utility. We believe that the findings of our pilot study are sufficiently positive to warrant further evaluation of the tool in a larger prospective study.

\section{Conclusion}

In conclusion, we have developed a novel values history tool, enhanced with graphics, whose aim is to support better ACP between patients and their future SDMs. Because of the rigorous development process, we believe the tool has face and 
content validity. Although early findings from our evaluation are promising, due to the small sample size and single centre nature of our study, we believe that further testing of the tool is warranted to support its more widespread adoption in clinical practice.

\section{Declarations}

This study received full approval from the Joint Research Ethics Board (reference number13-038-BP). All study participants gave written informed consent.

\section{Competing Interests}

The authors declare that they have no competing interests.

\section{Funding}

This was an unfunded study.

\section{Authors' Contributions}

PA contributed to the conception and design of the study, and the acquisition of the data. PA and JY contributed to the analysis and interpretation of the data, drafting of the manuscript, and give final approval of the version to be published. PA and JY agree to be accountable for all aspects of the work.

\section{Acknowledgements}

The authors thank: medical students Brianna Alkenbrack and Kaitlin Baenziger, University of Toronto, for their assistance in conducting this study. Thanks are also due to Nicole Devost, Palliative Care, Kavita Sridhar, Intensivist, and Lisa Huzel, Intensivist (all at Lakeridge Health, Oshawa, Ontario, Canada) for their assistance in the development and evaluation of the prototype tool. At Bridgepoint Health, we are grateful to Bridget Lauricella, Communicative Disorder Assistant and Robin Mowforth, Communicative Disorder Assistant for assistance with graphics; to Jan Kraus, Manager of Spiritual Care, for assistance with beta testing and graphics. Dr. Kira Payne and Ben Walmsley reviewed several versions of the GVHT and Barbara Mains provided assistance in editing.

\section{References}

1. Heyland DK, Barwich D, Pichora D, Dodek P, Lamontagne F, You JJ, et al. Failure to engage seriously ill hospitalized patients and their families in advance care planning: results of a multicenter prospective study. JAMA Intern Med 2013;173:778-87.

2. The SUPPORT Principal Investigators. A controlled trial to improve care for seriously ill hospitalized patients. The study to understand prognoses and preferences for outcomes and risks of treatments (SUPPORT). JAMA 1995;274:1591-8.

3. Heyland DK, Cook DJ, Rocker GM, et al. Defining priorities for improving end-of-life care in Canada. CMAJ 2010;182:E747-E752.
4. Sinuff T, Dodek P, You JJ, et al. Improving end-of-life communication and decision making: the development of a conceptual framework and quality indicators. J Pain Symptom Manage 2015;49:1070-80.

5. Sudore RL, Lum HD, You JJ, et al. Defining advance care planning for adults: a consensus definition from a multidisciplinary delphi panel. J Pain Symptom Manage 2017;53:821-32.e1.

6. Law Commission of Ontario. Improving the Last Stages of Life: Discussion Paper. Toronto, May 2017. Available at: http://www.lco-cdo.org/wp-content/ uploads/2017/05/LCO-Last-Stages-of-Life-Discussion-Paper-1.pdf. Accessed 27 Jan 2018.

7. International Patient Decision Aids Collaboration. IPDAS 2005: Criteria for Judging the Quality of Patient Decision Aids, 2006. Available at: http://ipdas .ohri.ca/ipdas_checklist.pdf. Accessed 30 Aug 2018.

8. Bernacki, RE., Block SD. Communication about serious illness care goals: a review and synthesis of best practices. JAMA Internal Med 2014;174:1994-2003.

9. College of Physicians and Surgeons of Ontario. Policy Statement \#6-16 Planning for and Providing Quality End-of-Life Care. Toronto, May 2016. Available at: http://www.cpso.on.ca/cpso/media/documents/policies/policyitems/end-of-life.pdf. Accessed 27 Jan 2018.

10. Shalowitz DI, Garrett-Mayer E, Wendler D. The accuracy of surrogate decision makers: a systematic review. Arch Intern Med 2006;166:493-7.

11. You JJ, Dodek P, Lamontagne F, Downar J, Sinuff T, Jiang X, et al. What really matters in end-of-life discussions? Perspectives of patients in hospital with serious illness and their families. CMAJ 2014;186:E679-87.

12. Heyland DK, Heyland R, Dodek P,et al. Discordance between patients' stated values and treatment preferences for end-of-life care: results of a multicentre survey. BMJ Support Palliat Care 2017;7:292-9.

13. Justin RG. The value history: A necessary family document. Theor Med 1987;8:275-82

14. Abhyankar P, Bekker HL, Summers BA, Velikova G. Why values elicitation techniques enable people to make informed decision about cancer trial participation. Health Expect 2011;14 Suppl 1:20-32.

15. Scheunemann LP, Arnold RM, White DB. The facilitated values history: helping surrogates make authentic decisions for incapacitated patients with advanced illness. Am J Resp Crit Care Med 2012;186:480-6.

16. Doukas DJ, McCullough LB. The values history. The evaluation of the patient's values and advance directives. J Fam Pract 1991;32:145-53.

17. Lambert P, Gibson JM, Nathanson P. The values history: an innovation in surrogate medical decision-making. Law Med Health Care 1990;18:202-12.

18. American Bar Association Commission on Law and Aging. Consumer's Tool Kit for Health Care Advance Care Planning: Tool \#2. Are some conditions worse than death? Second edition. Washington, DC; 2005. Available at: http://vaelderlaw.com/global_pictures/consumer_tool_kit_bk.pdf. Accessed 27 Jan 2018.

19. Fagerlin A, Pignone M, Abhyankar P, et al. Clarifying values: an updated review. BMC Med Inform Decis Mak 2013;13 Suppl 2:S8.

20. Witteman HO, Scherer LD, Gavaruzzi T, et al. Design features of explicit values clarification methods: a systematic review. Med Decis Making 2016;36:453-71.

21. Canadian Hospice Palliative Care Association. Advance Care Planning in Canada: National Framework. Ottawa, January 2012. Available at: http://www.advancecareplanning.ca/wp-content/uploads/2016/08/ACPFramework-2012-ENG.pdf. Accessed 27 Jan 2018.

22. Heyland DK, Pichora D, Dodek P, et al. The development and validation of a questionnaire to audit advance care planning. J Palliative Care Med 2012;2:119.

23. Detering KM, Hancock AD, Reade MC, Silvester W. The impact of advance care planning on end of life care in elderly patients: randomised controlled trial. BMJ 2010;340:c1345.

24. Wright AA, Zhang B, Ray A, et al. Associations between end-of-life discussions, patient mental health, medical care near death, and caregiver bereavement adjustment. JAMA 2008;300:1665-73. 